\title{
Einwirkung des Pilokarpins und Atropins auf den Zuckergehalt der Galle des Kaninchens.
}

\author{
Von \\ Sozo Hirayama. \\ (平山宗造) \\ (Aus dem Physiologischen Institute von Prof. Y. Sataké, \\ Tôhoku Universität $\approx u$ Sendai.)
}

Im Zusammenhange mit den Untersuchungen über den Einfluss des Phlorhizins auf den Zuckergehalt der Verdauungssäfte in der früheren und der vorangehenden Mitteilung, liess es sich durch eine Reihe von Experimenten ferner feststellen, ob parasympathische Gifte irgend einen Einfluss auf den Zuckergehalt der Galle ausüben oder nicht. Vorliegende Zeilen sollen seine kurze Skizze der diesbezüglichen Versuchsresultate geben.

Nur münnliche Kaninchen wurden verwandt. Was die Methodik betrifft, die in vorliegender Untersuchung erforderlich war, so verweise ich auf das bei früherer Gelegenheit Gesagte. ${ }^{1)}$

Das Kaninchen wurde auf dem Operationstische in Rückenlage gebunden, die Bauchhöhle längs der Linea alba aufgemacht, eine Glaskanüle mit einem Gummischlauch in den Ductus choledochus eingeführt und zubunden, und bald darauf die Bauchhöhle zugemacht. Das Tier blieb dann noch weiter auf dem Operationstisch gebunden die ganze Versuchsdauer hindurch. Demnach war die Fesselungs- sowie Operationshyerglyämie unumgänglich. Deshalb waren zunächst die diesbezüglichen Kontrollversuche vorzunehmen. Narkotika kamen niemals zum Gebrauch. Von der Benützung beiderseitig splanchnektomierter Tiere wurde es diesmal nur deswegen abgesehen, weil das so operierte Tier durch eine Behandlung wie in den vorliegenden Untersuchungen nicht selten schon frühzeitig stirbt.

1) So. Hir y a ma, Tóboku J. Exp. Med., 1924, 4, 507. 
Atropinum sulfuricum, in 0,1 prozentiger Lösung, wurde in einer Dose von $1 \mathrm{mg}$ pro $\mathrm{Kg}$ Körpergewicht intravenös eingeführt. Pilocarpinum hydrochloricum, in derselben Konzentration, wurde auch intravenös verabreicht. Jedes Tier erhielt $1 \mathrm{ccm}$ derselben Iösung zwei oder drei Male während des Versuchs.

Die Versuchsresultate lassen sich in nahestehenden drei Tabellen zusammenfassen.

Was die Versuchsergebnisse der Blutzuckeruntersuchung betrifft, so bieten sie nichts Aussergewöhnliches.

Bei den 8 Kontrollversuchen stieg der Blutzuckergehalt, der vor der Fesselung 0,08$0,14 \%$, durchschnittlich $0,11 \%$ betrug, im Laufe von etwa sechs Stunden, und zwar erreichte er sein Maximum in etwa 2-3 Stunden nach der Fesselung und Operation. Die maximalen Werte waren $0,14-0,23 \% o ́$, im Durchschnitt $0,19 \%$. Glykosurie trat ebenfalls auf ; bei zwei Fällen war sie aber nur geringfügig. Bei den 10 Atropinversuchen betrugen die Werte $0,08-0,12 \%$, durchschnitulich $0,09 \%$ resp. $0,11-0,22 \%$, im Mittel $0,18 \%$. In einigen Fällen war der Zeitpunkt, wo der Blutzucker seinen böchsten Gebalt zeigte, etwas verzögert. Beim einem einzigen Falle trat keine Hyperglykïmie auf. Der Harnzuckergehalt war auch bedeutend geringer gegenüber den Kontrollversuchen. Bei den 11 Pilokarpinversuchen war die Blutzuckererhöhung viel ausgeprägter gegenuiber den Kontrolltieren. Er stieg ron $0,1 \%(0,08-0,13 \%)$ bis auf $0,31 \%(0,22-0,49 \%)$. Ebenso verhielt sich auch der Harnzuckergehalt. Die Hyperglykämie und Glykosurie bei den Pilokarpinkaninchen in der früheren Mitteilung waren auch von derselben Stïke.1)

Auf die Sekretiongeschwindigkeit der Galle schien Pilokarpin einen schwach hemmenden Einfluss auszuäben, während Atropin ohne deutliche Einwirkung darauf blieb.

Die Reduktionskraft der Galle betrug bei den Kontrollversuchen etwa 0,01-0,02\%, und zwar war sie etwas höher während 2-3 Stunden nach der Operation und liess dann einigermassen nach. Die für die Zeiteinheit ausgeschiedene Zuckermenge ging parallel zur Konzentration, denn die Gallenausscheidungsgeschwindigkeit während des ganzen Versuchs war fast gar keinen Schwankungen unterworfen.

Auf das Reduktionsvermögen bewirkte Atropin eher verringernd, und infolgedessen war die für die Zeiteinheit ausgeschiedene Zuckermenge ebenfills vermindert.

Nach Atropindarreichung bei einem Kaninchen sah Neubauer die Zuckerreaktion der Galle verschwinden, die vor der Vergiftung nachgewiesen worden war. Er konnte sonst niemals an normalen sowie an mit Pilokarpin oder Atropin vergifteten Kaninchen Zucker in der Galle finden. Der Fehling schen Reaktion bediente er sich dabei.2?

Der Vermutung entsprechend war der Ausfall der Pilokarpinversu-

2) E. Neubauer, Biochem. Ztschr., 1920, 109, 82 . 
Tabelle

Kontrollver

\begin{tabular}{|c|c|c|c|c|c|c|c|c|c|c|c|c|}
\hline \multirow{3}{*}{$\mathrm{Nr}$. } & \multirow{3}{*}{\multicolumn{2}{|c|}{ Datum }} & \multirow{3}{*}{ 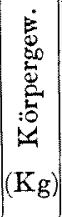 } & & & & & & & & & \\
\hline & & & & \multicolumn{2}{|c|}{$\begin{array}{l}\text { Blutzucker } \\
(\%)\end{array}$} & \multirow{2}{*}{$\begin{array}{c}\text { Harn } \\
\text { zucker } \\
(\%) \\
\text { Max. } \\
\text { n. } \\
\text { Op. }\end{array}$} & \multicolumn{6}{|c|}{ Menge ( $\mathrm{cm}$ pro St.) } \\
\hline & & & & $\begin{array}{l}\text { V.d. } \\
\text { Op. }\end{array}$ & $\begin{array}{c}\text { Max. } \\
\text { n. } \\
\text { Op. }\end{array}$ & & I St. & II St. & III St. IV & St. & V St. & VI St. \\
\hline 1. & 6. & III. '24. & 1,8 & 0,11 & 0,21 & 1,200 & 8,2 & 8,6 & 8,4 & 9,0 & 8,3 & 8,0 \\
\hline 2. & 13. & III. " & 1,5 & 0,10 & 0,18 & 1,200 & 9,5 & 10,0 & 10,8 & 11,8 & 10,7 & 8,8 \\
\hline 3. & 22. & III. " & 2,0 & 0,12 & 0,22 & & 5,8 & 6,0 & 6,4 & 5,6 & 5,4 & 5,2 \\
\hline 4. & 17. & V. , & 1,6 & 0,14 & 0,23 & 4,072 & 8,6 & 6,7 & 6,5 & 6,4 & 6,3 & 6,1 \\
\hline 5. & 11. & VI. " & 1,5 & 0,08 & 0,18 & 0,210 & 4,7 & 3,6 & 4,3 & 3,4 & 4,0 & 3,8 \\
\hline 6. & 21. & VI. " & 1,6 & 0,08 & 0,14 & 0,217 & 7,6 & 6,7 & 7,0 & 6,0 & 7,0 & 6,7 \\
\hline 7. & 25. & VI. ", & 1,7 & 0,12 & 0,21 & 2,800 & 5 & 6,0 & 9,4 & 7,2 & 5,4 & 5,6 \\
\hline 8. & 27. & VI. & 1,6 & 0,10 & 0,16 & & 5 & 5,5 & 6,4 & 6,3 & 5,9 & 5,9 \\
\hline
\end{tabular}

Tabelle

Atropinver

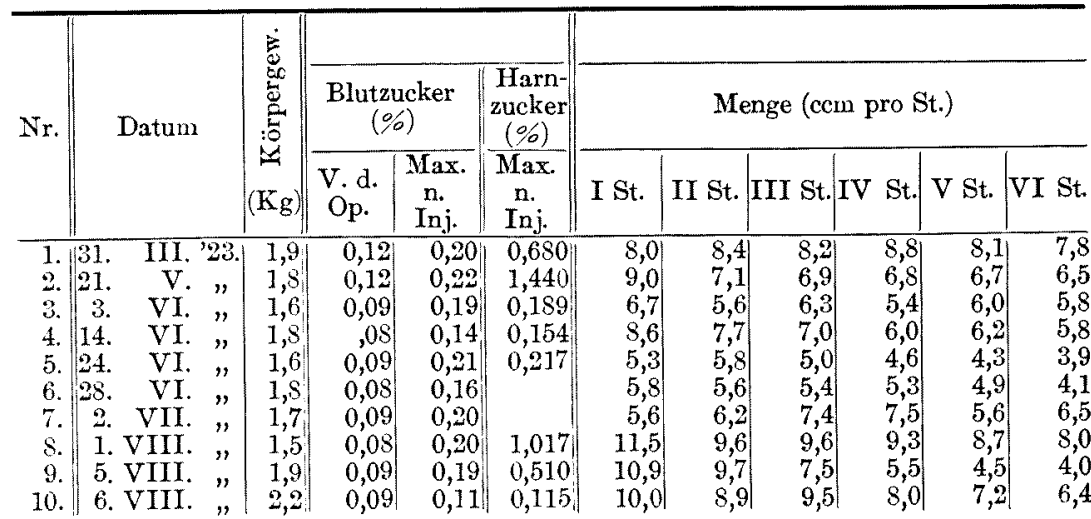

Tabelle

Pilokarpinver

\begin{tabular}{|c|c|c|c|c|c|c|c|c|c|c|c|c|}
\hline \multirow{3}{*}{ Nr. } & \multirow{3}{*}{ Datum } & \multirow{3}{*}{ 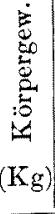 } & & & & & & & & & & \\
\hline & & & \multicolumn{2}{|c|}{$\begin{array}{c}\text { Blutzucker } \\
(\% \circ)\end{array}$} & \multirow{2}{*}{$\left|\begin{array}{c}\text { Harn } \\
\text { zucker } \\
(\%) \\
\text { Max. } \\
\text { In. }\end{array}\right|$} & \multicolumn{7}{|c|}{ Menge (cem pro St.) } \\
\hline & & & $\begin{array}{l}\text { V.d. } \\
\text { Op. }\end{array}$ & $\begin{array}{l}\text { Max. } \\
\text { n. } \\
\text { Inj. }\end{array}$ & & I St. & II S & & III St.|IV & St. & V St. & VI St. \\
\hline 1. & VI. '23. & 2,2 & 0,09 & 0,27 & 4,195 & & 11,9 & & 8,8 & & $2,3(1$ & $\left.1 \frac{1}{2} \mathrm{St}.\right)$ \\
\hline 2. & 3. VIII. , & 2,4 & 0,10 & 0,30 & 3,980 & & 9,3 & & 5,6 & & & \\
\hline 3. & 12. $\mathrm{X}$. & 1,6 & 0,11 & 0,31 & 1,020 & & 5,0 & & 10,0 & & & \\
\hline 4. & $\mathrm{X}$ & 1,9 & 0,12 & 0,34 & 1,018 & & 1,5 & & 2,2 & & & \\
\hline 5. & 1. XII. & 2,0 & 0,10 & 0,22 & 0,430 & & 2,7 & & 3,2 & & & \\
\hline 6. & I. 24 . & 2,0 & 0,08 & 0,32 & 1,400 & & 9,5 & & 5,7 & & $4,3(1$ & $\left.1 \frac{1}{2} \mathrm{St}.\right)$ \\
\hline 7. & II. ", & 1,7 & 0,11 & 0,36 & 7,414 & 10,0 & & 7,7 & 10,5 & 9,9 & 9,5 & 8,7 \\
\hline 8. & 18. & 1,6 & 0,09 & 0,29 & 4,263 & & 11,0 & & 5,5 & 5 & 2,3 & 2,4 \\
\hline 9. & II. " & 1,5 & 0,13 & 0,49 & 7,252 & 9,7 & & 9,1 & 9,2 & 8 & 4,9 & 3,9 \\
\hline 10. & 29. VII. " & 1,7 & 0,08 & 0,23 & 2,100 & 9,5 & & 8,7 & 8,4 & 6 & 4,8 & 4,2 \\
\hline 11. & 131. VII. & 1,8 & 0,09 & 0,25 & 4,510 & 6,1 & & 5,6 & 5,0 & & 2,7 & \\
\hline
\end{tabular}


I.

suche.

\begin{tabular}{|c|c|c|c|c|c|c|c|c|c|c|c|}
\hline \multicolumn{12}{|c|}{ Galle } \\
\hline \multicolumn{6}{|c|}{ Zucker $(\%)$} & \multicolumn{6}{|c|}{ Zucker (mg pro St.) } \\
\hline I St. & II St. & III St. & IV St. & V St. & VI St. & I St. & II St. & III St. & IV St. & V St. & VI St \\
\hline 0,21 & 0,20 & 0,17 & 0,12 & 0,10 & 0,09 & 1,72 & 1,72 & 1,43 & 1,08 & 0,83 & 0,72 \\
\hline 0,18 & 0,13 & 0,17 & 0,11 & 0,09 & 0,13 & 1,71 & 1,30 & 1,84 & 1,30 & 0,96 & 1,14 \\
\hline 0,20 & 0,21 & 0,18 & 0,16 & 0,12 & 0,08 & 1,16 & 1,26 & 1,15 & 0,90 & 0,65 & 0,42 \\
\hline 0,42 & 0,65 & 0,51 & 0,14 & 0,18 & 0,15 & 3,61 & 4,35 & 3,31 & 0,90 & 1,13 & $0,9]$ \\
\hline 0,11 & 0,15 & 0,12 & 0,14 & 0,12 & 0,12 & 0,52 & 0,54 & 0,52 & 0,48 & 0,48 & 0,46 \\
\hline 0,11 & 0,12 & 0,05 & 0,07 & 0,08 & 0,08 & 0,84 & 0,80 & 0,35 & 0,42 & 0,56 & 0,54 \\
\hline 0,10 & 0,09 & 0,09 & 0,09 & 0,06 & 0,06 & 0,51 & 0,54 & 0,85 & 0,65 & 0,32 & 0,34 \\
\hline 0,18 & 0,18 & 0,16 & 0,12 & 0,13 & $0,1.1$ & 1,03 & 0,99 & 1,02 & 0,76 & 0,77 & 0,6 \\
\hline
\end{tabular}

II.

suche.

Galle

\begin{tabular}{|c|c|c|c|c|c|c|c|c|c|c|c|}
\hline \multicolumn{6}{|c|}{ Zucker $(\%)$} & \multicolumn{6}{|c|}{ Zucker (mg pro St.) } \\
\hline I St. & II St. & III St. & IV St. & V St. & VI St. & I St. & II St. & III St. & IV St. & V St. & VI St. \\
\hline 0,23 & 0,22 & 0,17 & 0,13 & 0,10 & 0,09 & 1,84 & 1,85 & 1,40 & 1,14 & 0,81 & $\overline{0,70}$ \\
\hline 0,32 & 0,26 & 0,24 & 0,22 & 0,17 & 0,16 & 2,88 & 1,85 & 1,66 & 1,50 & 1,14 & 1,04 \\
\hline 0,15 & 0,12 & 0,1 & 0,14 & 0,12 & 0,11 & 1,00 & 0,67 & 0,76 & 0,76 & 0,72 & 0,64 \\
\hline 0,11 & 0,11 & 0,12 & 0,12 & 0,09 & 0,07 & 0,95 & 0,85 & 0,84 & 0,72 & 0,56 & 0,41 \\
\hline 0,13 & 0,11 & 0,10 & 0,09 & 0,09 & 0,09 & 0,69 & 0,64 & 0,50 & 0,41 & 0,39 & 0,31 \\
\hline 0,11 & 0,10 & 0,08 & 0,07 & 0,07 & 0,08 & 0,64 & 0,56 & 0,43 & 0,37 & 0,34 & 0,38 \\
\hline 0,20 & 0,17 & 0,10 & 0,09 & 0,10 & 0,07 & 1,12 & 1,05 & 0,74 & 0,67 & 0,56 & 0,45 \\
\hline 0,17 & 0,09 & 0,0 & 0,05 & 0,05 & 0,05 & 1,95 & 0,86 & 0,48 & 0,46 & 0,43 & 0,40 \\
\hline 0,26 & 0,17 & 0,12 & 0,10 & 0,10 & 0,11 & 2,80 & $1,6^{5}$ & 0,80 & 0,55 & 0,45 & 0,44 \\
\hline 0,11 & 0,08 & 0,0 & 0,09 & 0,07 & 0,07 & 1,10 & 0,71 & 0,85 & 0,72 & 0,50 & 0,45 \\
\hline
\end{tabular}

III.

suche.

Galle

\begin{tabular}{|c|c|c|c|c|c|c|c|c|c|c|c|}
\hline \multicolumn{6}{|c|}{ Zucker $(\%)$} & \multicolumn{6}{|c|}{ Zucker (mg pro St.) } \\
\hline I St. & II St. & III St. & IV St & V St. & VI St. & I St. & II St. & III St. & IV St. & V St. & VI St. \\
\hline & \multicolumn{2}{|c|}{0,35} & \multicolumn{2}{|c|}{$0,61\left(1 \frac{1}{1} \mathrm{St}.\right)$} & \multicolumn{2}{|c|}{4,90} & \multicolumn{2}{|c|}{1,54} & \multicolumn{2}{|c|}{0,93 (1 $\left.\frac{1}{2} \mathrm{St}.\right)$} \\
\hline \multicolumn{2}{|c|}{0,18} & \multicolumn{2}{|c|}{0,24} & & & \multirow{2}{*}{\multicolumn{2}{|c|}{0,82}} & \multirow{2}{*}{\multicolumn{2}{|c|}{0,67}} & & \\
\hline \multicolumn{2}{|c|}{0,35} & \multicolumn{2}{|c|}{0,3} & & & 2,62 & & & & & \\
\hline \multirow{2}{*}{\multicolumn{2}{|c|}{0,40}} & \multicolumn{2}{|c|}{0,41} & & & \multirow{2}{*}{\multicolumn{2}{|c|}{0,30}} & $0,4 \%$ & 1,75 & & \\
\hline & & \multirow{2}{*}{\multicolumn{2}{|c|}{$\begin{array}{l}0,92 \\
081\end{array}$}} & & & & & \multirow{2}{*}{\multicolumn{2}{|c|}{$\begin{array}{l}1,47 \\
2,27\end{array}$}} & & \\
\hline \multicolumn{2}{|c|}{068} & & & $0,76($ & $\left.1_{1}^{1} \mathrm{St}.\right)$ & \multicolumn{2}{|c|}{3,20} & & & $1,60($ & $\left.1 \frac{1}{2} \mathrm{St}.\right)$ \\
\hline 0,2$]$ & 0,3 & 0,18 & 0,1 & 0,10 & 0,11 & 2,10 & 2,31 & 1,89 & 1,84 & 0,95 & 0,96 \\
\hline & 76 & 0,6 & 0,4 & 0,33 & 0,37 & & 18 & 3,40 & 2,50 & 0,76 & 0,89 \\
\hline 0,3 & 0,7 & 0,4 & 0,4 & 0,33 & 0,83 & 3,6 & 7,01 & 4,14 & 3,86 & 1,62 & 1,29 \\
\hline 0,3 & 0,3 & 0,30 & 0,2 & 0,24 & 0,18 & 3,3 & 3,04 & 2,52 & 1,76 & 1,15 & 0,76 \\
\hline 0,3 & 0,8 & 0,28 & 0,2 & 0,18 & 0,14 & 1,8 & 1,85 & 1,40 & 0,91 & 0,49 & 0,35 \\
\hline
\end{tabular}


che. Die Reduktionskraft war während der ganzen Versuchsstunden ziemlich deutlich vermehrt. Folglich war, trotz der Verminderung der Ausscheidungsgesehwindigkeit in den späteren Stunden, die für die Zeiteinheit sezernierte Zuckermenge auch etwas vergrössert.

In der frïheren Mitteilung betrug der Zuckergehalt der Galle der Pilokarpin-Kaninchen $0,058 \%(0,012-0,108 \%)$, also nur ein bisschen mehr als der in der vorliegenden. Bei den doppelseitig splanchnektomierten Pilokarpin-Kaninchen in jener Mitteilung betrug er $0,039 \%$ ó $(0,023-0,059 \% 6)$.

In den anderen Versuchsreihen wollte ich die Einwirkung des Adrenalins sowie der Äthernarkose auf den Zuckergehalt der Galle studieren. Aus anderen Gründen liess ich dabei auch Pilokarpin mitwirken. Wenn irgend eine deutliche Differenz da wïre, sei es denn eine Vermehrung oder eine Verminderung, so könnte man etwas Bestimmtes über die Wirkungsweise ron Adrenalin oder $\ddot{A}$ ther auf den Zuckergehalt der Galle aussagen. Aber die Resultate zeigten keine Abweichung ron den Pilokarpinversuchen. Deshalb kann man ihnen keinerlei Bedeutung beilegen. Nur kann man Nicht-Vorhandensein eines hemmenden Einflusses des Adrenalins oder Athers auf die Pilokarpinglykocholie schliessen.

Aus Obigem geht hervor, dase Pilokarpin glykocholische Wirkung auf Kaninchen ausübt, während Atropin keinen Einfluss auf die Galle hinsichtlich der Menge und der Reduktionskraft besitzt. 